\title{
Descolonizar la historia de la tradición filosófica en Colombia: la necesidad de reevaluar los postulados historiográficos actuales ${ }^{*}$
}

Fecha de entrega: 24 de enero de 2020

Fecha de evaluación: 26 de febrero de 2020

Fecha de aprobación: 3 de marzo de 2020

Laura Patricia Bernal Ríos**

\section{Resumen}

Se ofrece una reflexión sobre los avances y las tendencias de discusión en la historia de la tradición filosófica en Colombia y sus presupuestos historiográficos, como un aporte al debate sobre la relevancia de pensar las tradiciones filosóficas nacionales en el siglo xxi. A partir de las categorías valorativas de las diferentes producciones intelectuales se determina si hacen parte o no de la tradición filosófica nacional. El concepto de "normalización filosófica" es analizado como categoría interpretativa de la tradición colombiana y se sugiere su obsolescencia dadas sus implicaciones; con el propósito de superar el reduccionismo, la exclusión y el sesgo que conlleva esta categoría. Se propone la reivindicación de los intentos de análisis social del siglo xix y de las producciones filosóficas regionales con el propósito de aportar a una

* Este artículo recoge algunas reflexiones de la investigación doctoral "Decolonizar la enseñanza de la filosofía en Colombia" del Doctorado en Educación de la Universidad de Caldas, Colombia. Citar como: Bernal Ríos, L. P. (2020). Descolonizar la historia de la tradición filosófica en Colombia: la necesidad de reevaluar los postulados historiográficos actuales. Cuadernos de Filosofía Latinoamericana, 41(123), 149-165. Dol: https://doi.org/10.15332/25005375/4497

** Profesional en Filosofía y Letras, Magister en Educación y estudiante del Doctorado en Educación de la Universidad de Caldas, Colombia. Integrante del grupo de investigación Maestros y Contextos y docente del Departamento de Estudios Educativos de esta misma institución. Correo electrónico: laura.bernal@ucaldas.edu.co 
reconstrucción decolonial, amplia y sin sesgos de la historia de la tradición de la filosofía colombiana.

Palabras clave: filosofía colombiana, normalización filosófica, filosofía regional, historia de la filosofía en Colombia, reconstrucción decolonial.

\section{Decolonizing the History of the Colombian Philosophical Tradition: The Need to Reassess Current Historiographic Postulates}

\section{Abstract}

This article is a reflection on the progress and discussion trends of the history of the Colombian philosophical tradition and its historiographic presuppositions, and aims to contribute to the debate about the relevance of thinking about national philosophical traditions in the twenty-first century. Based on the valuation categories of the different intellectual products, it can be determined whether or not they are part of the national philosophical tradition. The concept of "philosophical normalization" is analyzed as an interpretative category of the Colombian tradition and, given its implications, its obsolescence is suggested with the aim of overcoming the reductionism, exclusion, and bias entailed by it. This article vindicates the nineteenth century attempts at social analysis and regional philosophical productions with the aim of contributing to a decolonial, broad, and unbiased reconstruction of the history of the Colombian philosophical tradition.

Keywords: Colombian Philosophy, Philosophical Normalization, Regional Philosophy, History of Philosophy in Colombia, Decolonial Reconstruction.

\section{Pensar tradiciones nacionales en el siglo XXI}

Después de dos siglos de la Independencia de la Corona española, Colombia no termina de consolidarse como un Estado nacional. No lo logró en los periodos de mayor 
acogida mundial de la democracia, cuando el Estado todavía tenía el papel de garante del bienestar y la paz social. Ahora, en el siglo xxi, con el paulatino debilitamiento de los Estados nacionales de los sures globales y el éxito de la contrarreforma neoliberal, puede ponerse en tela de juicio la insistencia en los debates que buscan la definición de identidades nacionales, tradiciones locales o pasados colectivos. Hablar entonces de si existe o no tradición filosófica en Colombia parece un problema vacuo, una tarea inútil propia del siglo pasado. Sin embargo, el objetivo de este artículo es argumentar todo lo contrario.

No se trata de plantear resistencias culturales e intelectuales locales ante el expansivo proceso de homogenización neoliberal, en una especie de gesto nacionalista anacrónico. En su lugar, se trata de constatar que por mucho que el mercado global posea un proyecto cultural que consiste en derribar las particularidades locales para facilitar su expansión, las sociedades no alcanzarán nunca una total homogenización. Se trata de no caer en la falacia de ignorar la necesidad de pensar nuestros fenómenos sociales permeados por la experiencia colonial, e inscritos, por supuesto, en este contexto global. Por tanto, la respuesta es afirmativa: Colombia, como grupo social definido a partir de un proyecto nacional que lleva ya más de doscientos años, debe reafirmar sus tradiciones nacionales, como prerrogativa para conservar y fortalecer la cohesión social.

Ahora bien, se da por sentada la necesidad de reconstruir nuestro pasado intelectual y, en lo que respecta al interés de este artículo, de la filosofía. En este marco surge el debate en torno a los siguientes interrogantes: ¿existe realmente una tradición filosófica en Colombia?, ¿cómo se han comprendido el desarrollo y la evolución de la tradición filosófica en el contexto colombiano?, ¿qué sesgos o aporías se pueden identificar en la manera como se ha normalizado la historia de la filosofía en el caso colombiano? $\mathrm{Al}$ explorar respuestas se hallan varias posturas, unas radicales, que afirman que no existe tradición filosófica en Colombia por la simple comprobación de su baja calidad y poco impacto e incidencia dentro de la tradición más amplia de Occidente; y, otras, que consideran que si bien se debe aceptar su posición marginal, subalterna y su poca o nula influencia en el canon filosófico occidental, siempre han existido intentos de reflexión sobre la realidad social del país, lo cual, dentro un concepto más amplio de filosofía, se puede considerar como acontecimiento filosófico. Más allá de centrar la reflexión en este aspecto, el problema radica en cómo construir la historia de la tradición filosófica en Colombia, y bajo cuáles presupuestos y postulados se construye dicha historia. 
En este artículo se busca evaluar cuáles han sido los diferentes componentes, elementos interpretativos y posturas sobre los cuales se ha construido la historiografía de la filosofía colombiana. Para tal fin, se revisan trabajos y autores que se han planteado este conjunto de cuestiones: ¿cómo se ha concebido la historia del desarrollo de la tradición filosófica en Colombia? ¿Bajo qué categorías se deben valorar las diferentes producciones intelectuales para determinar si hacen parte o no de una tradición filosófica nacional? Así, con base en la revisión de los trabajos de Jaime Jaramillo Uribe (1954; 1977), René Campis (2006), Juan Camilo Betancur (2015), Carlos Arturo López (2012; 2014) y Damián Pachón (2011; 2019), el análisis partirá de la categoría de "normalización filosófica", que ha sido usada para establecer periodizaciones y explicar el proceso de ruptura del pensamiento colombiano. Sin embargo, esta categoría ha generado una condición excluyente, en la medida en que concibe a la tradición filosófica como un proyecto monocultural. Por lo tanto, es imprescindible replantear su uso para llegar a una historia más amplia que pueda describir mejor la complejidad del proceso de formación de la tradición filosófica colombiana, que abarcaría más momentos, generaciones y autores. Así, se puede concebir la categoría de tradición como un concepto que implica cambio, reemplazo y sucesión; como una categoría compuesta y compleja para una reevaluación y reconstrucción en clave decolonial de la historiografía de la filosofía en Colombia.

\section{La "normalización filosófica" como categoría interpretativa de la tradición colombiana}

En cualquier estudio historiográfico surge la necesidad de hacer periodizaciones para dar cuenta de los diferentes momentos en el desarrollo de una tradición. En su construcción se suele prestar atención a generaciones, esfuerzos individuales o fenómenos sociales más generales, a partir de los cuales se realizan estas delimitaciones temporales. En el caso de la historia de la filosofía en Colombia, el concepto de "normalización filosófica" fue adoptado por los historiadores a partir de la década de los setenta del siglo xx, para describir un momento concreto que se concibió como el gran paradigma de la producción filosófica en el país y, por lo tanto, como el eje central para entender el desarrollo de esta disciplina. Al respecto del origen de este concepto encontramos que:

El término "normalización" proviene del concepto de "normalidad filosófica", usado en el contexto más general de Latinoamérica, desde que Francisco Romero 
lo acuñó en el segundo tercio del siglo xx. En años recientes, algunas críticas al concepto han mostrado los riesgos que entraña su uso irreflexivo. Raúl FornetBentacourt, por ejemplo, señala que la categoría de "normalidad filosófica" supone una determinada concepción de filosofía que no estamos obligados a compartir y que, más bien, implica varios sesgos para el estudio de la producción filosófica en Latinoamérica. [...] El concepto termina centrando la importancia de los "fundadores de la normalidad" en su aporte a la filosofía académica producida con la institucionalización universitaria. (Betancur, 2015, p. 139)

Esta categoría se adoptó en el país para describir cómo en la década del cuarenta, una generación concreta fundó la Facultad de Filosofía de la Universidad Nacional de Colombia, lo que consolidó el proyecto de institucionalización universitaria de la disciplina. Es decir, este concepto se refiere principalmente al cambio de los medios de hacer filosofía, y no solo a la entrada de la filosofía moderna en Colombia, la cual, como demuestran varios autores, aconteció décadas antes. Esto obedece a un proceso de institucionalización, a "la consolidación de la filosofía como oficio disciplinar que comenzó a gozar de autonomía temática e investigativa” (Betancur, 2015, p. 139). A continuación, una síntesis precisa de los tres rasgos característicos de esta categoría creada por Romero:

- La consideración de la filosofía como una forma de saber racional autorreflexivo y, por tanto, superior y "tardío" (de influencia hegeliana) en relación con otras manifestaciones culturales.

- La vinculación del quehacer filosófico con una actividad profesional cuyo ejercicio requiere justamente "cauces normales" que implican a su vez la inserción de la filosofía en los procesos de organización y de institucionalización de la cultura.

- La concepción de la filosofía como una vocación personal de conocer y estudiar la realidad, relacionada con un hábito de estudio y una historia particulares (Campis, 2006, p. 417).

Desde este punto de vista, se podría afirmar que esta categoría permite comprender una de las etapas de la tradición. Sin embargo, aquí nace parte del problema, porque la categoría en sí misma carga una manera de concebir la filosofía, a saber, la filosofía académica cultivada y reproducida en la universidad, comprendida y delimitada como 
una institución formal-formalizada. En este orden de ideas, el concepto de normalización no solo se usa para describir un momento de ruptura, sino que se erige como el ideal de filosofía y, en este sentido, todo lo que no esté dentro de sus márgenes formales-formalizados no clasifica como tal. Así, toda actividad del pensamiento que no se realice dentro de estos cánones de profesionalización, o dentro de un ambiente académico, carecería de valía, y, por ende, no haría parte de la tradición. Autores como Rubén Sierra, Guillermo Hoyos o Rubén Jaramillo reprodujeron esta postura, lo que generó un evidente y contraproducente sesgo. Esta circunstancia deviene en dos problemas: todo lo que venga después de este momento de normalización será excluido si no cuenta con el rigor de la academia. Segundo, y más preocupante aún, se negará la existencia de una rica producción intelectual anterior, la cual emergió amparada por otras condiciones históricas en las cuales no era posible esta clase profesionalización - verbigracia, la reflexión filosófica muchas veces estaba en conjunción con otras actividades, como la política-.

Pensar la filosofía bajo estos preceptos lleva a estos autores a afirmar que la filosofía colombiana nace hasta este momento. Pero lo que en realidad sucedió fue que una nueva generación buscó romper con una vieja, atrasada y dogmática tradición, heredada del siglo XIX, para alcanzar la tan anhelada filosofía moderna. Para tal fin era muy importante lograr esta autonomía bajo el amparo institucional, una característica propia de esta tradición eurocéntrica que se quería adoptar. Este propósito último de profesionalización de la filosofía es el único valor que admite una historiografía sesgada. Combatiendo este sesgo, autores como Betancur (2015), López (2012; 2014) y Pachón $(2011 ; 2019)$ aseguran que esta postura impide ver la complejidad de los procesos, al no aceptar el ejercicio filosófico presente en otros cánones de producción. El asunto es que quienes pensaron problemas relacionados con la sociedad, la religión, la política o el derecho - pero que no fueron profesores o filósofos profesionales, como lo indica el modelo europeo imitado en Colombia - fueron desdeñados de esta tradición y su cultivo se convierte en producción subalterna y periférica.

\section{Superando el colonialismo y la exclusión de la normalización filosófica}

Respecto a esta labor de exclusión, debemos determinar dos aspectos: 1) la filosofía académica —institucionalizada - es concebida como la única producción con valor 
filosófico, en este sentido, todo el pensamiento no institucionalizado no clasifica como filosofía. 2) La centralización de la producción académica y del cultivo intelectual generaron, en consecuencia, la subalternización de la producción y cultivo proveniente de las regiones donde no se contaba con instituciones académicas que soportaran el valor de los trabajos filosóficos.

Como respuesta a estos dos grandes sesgos, Betancur (2015) demuestra la existencia de una rica producción filosófica — muchas veces de carácter individual- que no se reduce a la normalización filosófica de la institución universitaria formalizada y los medios académicos de control de la producción, el cultivo y el quehacer filosófico. Incluso, se puede albergar una postura mucho más abierta, como la de Campis (2006), quien, con un criterio geográfico, pretende cerrar esta infructuosa discusión:

Por el solo hecho de que se hubiera estudiado, escrito, discutido — sea en el formato que sea, informal o formalmente- filosofía en el territorio geográfico que corresponde a la forma de organización política llamada Colombia, ya hay que reconocer que tenemos una tradición propiamente colombiana en filosofía, así no la hubieran reconocido Rubén Sierra, Cayetano Betancur y otros, y así no sea del mejor agrado de ellos, mío o suyo. (Campis, 2006, p. 414)

De igual manera, Jaramillo Uribe (1954) defiende una construcción más amplia de esta tradición, pero centrándose en la periodización del siglo XIX, en función de su idea de reconocer el trabajo de "nuestros padres del [siglo] xıx". Entonces, varios son los conceptos que se deben considerar aquí, antepuestos a las características de la categoría de "normalización”, a saber:

- Medios alternativos de producción: los normalizadores afirman que solo la filosofía formulada dentro de los muros académicos es verdadera filosofía, sin embargo, dentro de los procesos históricos del país, autores como Jaramillo Uribe han demostrado que el ejercicio de reflexión filosófica se da en muchos ámbitos de la vida pública, y no inicia cuando se fundan facultades de filosofía.

- Descentralización intelectual: Colombia es un país de una fuerte tradición centralista, no solo en el aspecto administrativo. Por considerar un ejemplo, Bogotá se ha convertido en el gran epicentro cultural e intelectual del país, a costa de negar la producción que se presenta en otras regiones. De hecho, regiones como Caldas, 
Antioquia o la Costa Atlántica han realizado significativos aportes, sin los cuales no se podría comprender esta "evolución” o, por lo menos, su trayectoria. Piénsese en Julio Enrique Blanco, Danilo Cruz Vélez o Fernando González.

- Filósofos subalternos: no solo el profesor de filosofía puede ser considerado filósofo, nuestro medio ha estado lleno de personalidades individuales que han legado una gran obra sin haberse ceñido a la rigurosidad académica.

Respecto al primer punto, encontramos la siguiente afirmación de Campis (2006):

Resulta nuevamente interesante y provocador lo planteado por Manuel Rodríguez en su libro sobre filosofía colombiana en el siglo $\mathrm{xx}$, donde sostiene que hubo algo así como un periodo en el que la filosofía colombiana o el pensamiento filosófico colombiano se exilió en la literatura, la crítica literaria, el periodismo y los manifiestos [...] Estos dos hechos solos, la tesis de la "filosofía en el exilio" y el caso de Julio Enrique Blanco, también documentado por Rodríguez en su libro, obligaría a reescribir nuestra historiografía en materia de filosofía colombiana del siglo xx. (Campis, 2006, p. 413)

Respecto al segundo punto, no es de extrañar que un país tan arraigado a la herencia hispana y colonial de la centralización del poder haya reproducido esta tendencia en los diferentes ámbitos sociales, incluidos los intelectuales. No es secreto que Bogotá ha tenido un papel tutelar en el destino el país, pero muchas veces ha desconocido el enorme aporte de las regiones. Según Campis:

[...] esta no es la única imprecisión en la versión de los historiadores tradicionales de la filosofía en Colombia [...] Solo así se explica que un público más amplio no haya podido reconocer los distintos desarrollos regionales y el que se asuma corrientemente en los círculos académicos de filosofía en el país que la modernidad comienza con el Instituto de Filosofía de la Universidad Nacional. (Campis, 2006, p. 413)

Se puede considerar, pues, que la problemática de la construcción historiográfica de la tradición filosófica colombiana radica en la adopción de una serie de categorías interpretativas que han generado una serie de prácticas coloniales y de exclusiones que han impedido abarcar todo el panorama intelectual del país, al establecer una serie 
de imprecisiones, vacíos y contradicciones. La narración de lo que López (2012) llama el mito de la "normalización filosófica" en Colombia impide la inclusión de las producciones filosóficas locales y regionales, que se estudian partiendo de los prejuicios heredados por la tradición filosófica europea. En general, estos tres aspectos expuestos son los resultados de la adopción de la categoría de "normalización". La historiografía actual ha buscado superar estos sesgos a partir de la reivindicación de producciones realizadas en otros medios intelectuales, producciones realizadas por filósofos que no hicieron parte de ninguna institucionalidad académica, así como la reivindicación de las producciones regionales. En palabras de López:

Buscamos afanosamente un espacio común donde la inercia de los modos hegemónicos de ser filósofo no se impongan sobre los materiales filosóficos no canónicos que nos interesan, donde otros tipos de actividad filosófica constitutivos de la filosofía misma pero silenciados por la forma en que se cuenta su historia, en que se establece un canon a partir de la idea de tradición, puedan ser investigados y apropiados sin olvidarlos o sin considerarlos como esfuerzos infructuosos, débiles o simples repeticiones de lo dicho en otro lugar. (López, 2012, p. 323)

Una cuestión que en cierto modo carece de un tratamiento explícito es el énfasis que la normalización filosófica de la tradición establece en el aspecto de "la producción filosófica", vista a la luz de las estructuras académicas institucionales, más allá del cultivo de la filosofía como una forma de habitar contextualizado y de quehacer cultural frente a las contingencias del mundo. Puede ser que el énfasis puesto en "la producción" se avenga con las exigencias ontológicas y de las estructuras sociales que parapetan la Modernidad europea, a la vez que resulta funcional a la imposición colonial y excluyente del saber. Todo ello, en detrimento de otras categorías evaluativas frente a las posibles y pensables tradiciones filosóficas, que consideran aquellas que no se reconocen con todos sus efectos en el canon eurocéntrico u occidental o que, de alguna manera, llegan a criticar sus rudimentos. Así pues, categorías como "cultivo del saber", que trascienden o proponen otras lógicas distintas a las formas de producción institucionalizadas implantadas en nuestra realidad colonial y excluyente, son desechadas a priori como formas de reconocimiento y construcción de tradiciones emergentes o históricamente invisibilizadas, como bien lo muestra el caso colombiano. No obstante, en esa tradición invisibilizada también podemos encontrar la semilla para 
deconstruir - o por lo menos para revaluar - la postura colonial y excluyente de los normalizadores (lo que denomino la reconstrucción decolonial).

\section{Reivindicación del siglo XIX: el valor de los primeros intentos de análisis social}

Superar el sesgo desproporcionado a favor de la "normalización filosófica" conlleva dos tareas: una hacia atrás y otra hacia adelante. Se debe recuperar todo lo que hubo antes de la fundación e institucionalización de las facultades de filosofía, y a su vez, todo lo que se produjo y cultivó después, pero fuera de sus muros. Los normalizadores radicales despertaron una serie de contradicciones imposibles de resolver cuando favorecieron el despropósito de descartar todo lo que estaba detrás, al despreciar nada más que un siglo entero de trabajo intelectual que, si bien no se ajustaba a la severidad de la universidad europea, fue fruto de la necesidad real de consolidar el proyecto de Estado nacional; así como de definir los diferentes imaginarios tanto políticos como culturales que darían dirección al destino de Colombia como nación independiente. A partir de este punto, Jaramillo Uribe - uno de los padres de la nueva historia en Colombia - ya observaba en la década de los cincuenta del siglo xx, la necesidad de considerar la filosofía como todo acontecimiento de pensamiento que busca entender las problemáticas sociales de las diferentes épocas del país. Lo anterior, guardadas las proporciones, resulta equiparable a rescatar hoy en día el cultivo de la filosofía como una forma de habitar en contexto y de quehacer cultural frente a las contingencias del mundo con base en las particularidades propias.

En este sentido, decir que en Colombia solo se empieza a hacer filosofía cuando esta labor se institucionaliza en un lugar y momento muy concretos, como lo es la Facultad de Filosofía de la Universidad Nacional en la década de los cuarenta, implica dar la espalda y olvidar una rica tradición que hunde sus raíces en el siglo xix. De hecho, en El pensamiento colombiano en el siglo XIX, Jaramillo Uribe (1982) pretende analizar, sintetizar y periodizar esta etapa, comprendida como el momento en que se sientan las bases del pensamiento filosófico en el país.

Jaramillo Uribe (1977) no desconoce que sea una tarea difícil debido a las condiciones de producción marcadas por el irrisorio y bajo impacto de la filosofía colombiana a nivel mundial, y, sobre todo, por su nula profesionalización en una época llena de 
inestabilidad social. Por lo tanto, se plantea entonces una cuestión cardinal: ¿vale la pena pensar en una tradición nacional aunque sea "marginal" y "subalterna"? La respuesta del autor es que sí; el intento de negar este desarrollo ignora, precisamente, el significado mismo del concepto de "tradición”.

Para que en un país exista una tradición de pensamiento filosófico no se requiere que la producción haya sido voluminosa en el número de escritos, ni que quienes hayan cultivado este tipo de saber brillen por su originalidad en la historia del espíritu. Lo indispensable es que, siempre, y en cada momento, un grupo de hombres cuya influencia se hace sentir en el contorno social, haya mantenido el contacto con el saber filosófico de su tiempo y con el saber filosófico del pasado [...] Del diálogo, crítica y negación de sus diferentes corrientes, vive y se hace fecundo el pensamiento. Cada etapa de su historia presupone otro antecedente, del cual parte toda nueva actividad, no importa, muchas veces, que dicho antecedente sea pobre o equivocado a juicio de quienes lo han recibido y se han hecho cargo de la misión de mantenerlo, de enriquecerlo y hacerlo más apto para explicar los enigmas del universo o para resolver los problemas de la vida espiritual propios de una cultura o de una generación. Desde este punto de vista es por lo que todo saber - y en primer lugar el saber filosófico- es un saber histórico que debe contar con lo que se hereda, es decir, con la tradición [...] Sirvan estas consideraciones generales para preparar la respuesta al interrogante de si tenemos en Colombia una tradición filosófica. Creemos que tal interrogante debe responderse afirmativamente. (Jaramillo Uribe, 1977, pp. 33-34)

Esta consideración es más que suficiente para reconocer la "tradición" como una categoría en sentido amplio; una que serviría para deconstruir —o por lo menos para revaluar - la postura colonial y excluyente de los normalizadores. Jaramillo Uribe realiza también una periodización de la evolución del pensamiento filosófico colombiano que puede resumirse así: 1) la escolástica heredada de la época colonial; 2) la influencia de la Ilustración, resumida en dos sistemas importantes: el liberal y el romanticismo político francés, que buscarán el pensamiento moderno europeo; y 3 ) un pensamiento que tiende al tradicionalismo y que fundamentará la ideología conservadora.

En resumen, cuando llega la Independencia, la Corona española había reducido la reflexión filosófica en el país a la escolástica; pero una vez inició la era republicana, 
se formaron dos grandes sistemas de pensamiento que se aglutinaron bajo los proyectos políticos del liberalismo y el conservadurismo. Dentro de estos entran muchos sistemas filosóficos europeos que son adoptados con entusiasmo, vistos como respuestas para resolver los problemas de organización social en el país. Y es a partir de dos figuras emblemáticas de este periodo, José María Samper y Miguel Antonio Caro, que se puede construir una breve descripción de estas tradiciones:

José María Samper fue un tipo representativo de la generación radical del siglo pasado: benthamista primero y spenceriano después en filosofía, individualista en política, fisiócrata y clásico en economía. Si ahora pasamos a una vertiente distinta de nuestra historia de las ideas, la representada por un tipo de mentalidad de fuerte ancestro hispánico, mezclado con influencias tomistas e ideas tradicionalistas de fuente inglesa y francesa, encontramos la gran figura de don Miguel Antonio Caro. (Jaramillo Uribe, 1954, p. 66)

En conclusión, el siglo XIx fue la época en que se forjaron las ideas constituyentes de la Nación, no solo en los campos político y económico, sino también en el cultural y en el intelectual. El trabajo de Jaramillo Uribe representa un verdadero acto de reconstruir una tradición nacional. Al mismo tiempo, autores como Camacho Roldán, José María Samper y Miguel Antonio Caro produjeron reflexiones concretas en el contexto de la confrontación ideológica en el periodo de consolidación de las instituciones del Estado, todas ellas encaminadas a entender los problemas del país y, en esa medida, se les debe considerar parte de la tradición filosófica en Colombia.

No se debe asignar un crédito excesivo al factor excluyente de la exigencia por la correspondencia (casi de veneración) con la tradición filosófica europea. Y es que muchos de quienes han puesto en entredicho la posible tradición filosófica colombiana, lo hacen apoyados en argumentos coloniales y eurocéntricos. De la clase de que si no producimos filósofos a imagen y semejanza de como lo hace la academia europea o angloamericana, entonces no tenemos filosofía. Pero, al mismo tiempo, se erige una fuerte exigencia de autenticidad a la hora de condenar a los autores del xIx por haber adoptado las corrientes filosóficas de la época, sin mediar un acto reflexivo. Pachón (2011) realiza una reivindicación de la historia de la tradición filosófica de Colombia desde la época de la Colonia hasta la actualidad en la cual establece la siguiente periodización: Colonia, Ilustración, siglo xix y Modernidad, en la cual incluye el olvido de la herencia filosófica del pasado colombiano y la crítica a la razón eurocéntrica en lo 
que llama "fetichización del pensamiento europeo" en la historia oficial de la filosofía en Colombia. Esto es suficiente para sugerir la reconstrucción decolonial.

\section{Para una reconstrucción decolonial de la historia de la filosofía en Colombia: la producción filosófica regional}

El segundo aspecto importante que debe reivindicarse para elaborar una auténtica historia de la filosofía en Colombia es la producción filosófica realizada fuera de Bogotá (la regionalización de la filosofía). Sin embargo, este tema nos remite a otro de primer orden: la entrada de la filosofía moderna a Colombia, que no se dio por la generación normalizadora en la capital, sino en Barranquilla, con la figura de Julio Enrique Blanco. Es decir, el sesgo centralista que se ha adoptado para interpretar la producción filosófica en Colombia ha imposibilitado hacer una reconstrucción acertada del momento de superación de la filosofía escolástica, de la comprensión contextualizada de las diferentes teorías liberales y conservadoras fuertemente politizadas, y de la introducción en el país de la filosofía moderna, representada por autores como Hegel o Nietzsche.

No considerar las producciones regionales ha ocasionado que muchos historiadores den por sentado que el estudio de la filosofía moderna inició en la capital, en la Facultad de Filosofía de la Universidad Nacional, y no en el puerto de Barranquilla, en donde la generación de la revista Voces, gracias al intercambio material y cultural propiciado por la ciudad portuaria, formó un proceso de renovación en diferentes disciplinas. Este acontecimiento se extendería a Bogotá, cuando muchos de sus integrantes coincidieron allí. Es decir, hasta en este punto están equivocados buena parte de los historiadores "normalizadores", porque si bien la renovación de muchas disciplinas se dio en la capital del país, como punto geográfico, quienes llevaron a cabo estos procesos provenían de diferentes regiones. Y no es poca cosa aclarar que antes de que esto sucediera, Bogotá se encontraba sumida en la atrofia cultural ocasionada por la mentalidad conservadora que regía los destinos del país a comienzos del siglo xx, luego de la llamada "Regeneración”.

Campis (2006) considera que la influencia de la generación de Voces nos lleva a replantear gran parte de la historia de la filosofía en Colombia. 
Un breve inventario de algunos de los autores tratados en los textos filosóficos publicados en Voces resulta suficiente para desmentir la idea aceptada por Cayetano Betancur, Rafael Carrillo, Danilo Cruz, Sierra, Mejía, Jaramillo, Hoyos y mucho otros, y que Gutiérrez Girardot expresaba líricamente así: "es de justicia reconocer que fueron Rafael Carrillo y Danilo Cruz Vélez quienes introdujeron la filosofía moderna en Colombia”. (Campis, 2006, p. 421)

Uno de los argumentos que Campis también desmiente es aquel que afirma que la figura solitaria de Blanco - en un contexto donde la filosofía no tenía un espacio social consolidado - fue ignorada, y, en consecuencia, no sería lícito sostener que haya ejercido una real influencia en filósofos posteriores. De ser esto cierto, el trabajo de Blanco cayó en el vacío y no contribuyó al desarrollo filosófico posterior. Pero Campis demuestra que sí hubo una difusión del pensamiento de Blanco: "El agravante para el caso de Blanco es que muchas de las personalidades más conocidas de la escena filosófica bogotana conocían de él y de su trabajo, ya que había publicado varios ensayos en revistas de Bogotá y Medellín” (Campis, 2006, p. 424). El descubrimiento del trabajo filosófico de Blanco ha abierto nuevas perspectivas en la historiografía de la filosofía en Colombia, una página importante en la reconstrucción del siglo xx en el país. Esta es una clara muestra de la validez de la descentralización y de la descolonización de la historia en Colombia, camino en el que todavía queda mucho por recorrer y en donde radican las semillas que este artículo se propone sembrar.

\section{A modo de conclusión}

Podemos identificar las categorías que han sido utilizadas para evaluar la tradición de la filosofía en Colombia, que en la mayoría de los casos han sido trasladadas e implantadas en el contexto colombiano desde otras tradiciones de manera irreflexiva, lo que imposibilita un estudio historiográfico más pertinente y completo. Dichas categorías de análisis se pueden resumir así:

- Autonomía: ninguna tradición se genera a sí misma en un espacio cerrado. La tradición colombiana pertenece a la tradición occidental, impuesta en un proceso de conquista y colonización. En este sentido, heredamos una tradición que rige los modos de pensamiento en el continente latinoamericano. Sin embargo, la autonomía solo se puede lograr si se reevalúan las bases de esta tradición y se busca una interpretación de nuestra realidad desde una perspectiva decolonial (lo que 
denomino reconstrucción colonial). De ninguna manera se logrará con un ejercicio de negación, basado en el argumento de que como no es europea, no merece ser considerada.

- Institucionalidad: "una verdadera filosofía solo es aquella que se da en el contexto de la institución universitaria”. De ninguna manera esta sentencia puede considerarse como una categoría de análisis acertada. De hecho, Jaramillo Uribe (1954) argumenta cómo el pensamiento germina en la libertad de su poder crítico, lo cual es restringido por la misma lógica de las instituciones. De manera que, si bien la filosofía debe aspirar a su institucionalización para adquirir una posición social más clara y fuerte, no debe, en ninguna medida, generar un sesgo e ignorar todo lo que se produce fuera de sus muros.

- Centralismo: es imprescindible evitar una visión monocultural para el análisis historiográfico de la filosofía en el país. Si bien existen epicentros que sobresalen por su alto desarrollo en diferentes disciplinas, no debe imponerse un modelo de caracterización excluyente bajo sus principios. La mayoría de historias (en cualquier ámbito) suelen darle una importancia vital a Bogotá, que ignora los fenómenos que se dan en otras regiones. Esto, además de ser excluyente, impide un análisis acertado de los procesos sociales, como se pudo comprobar en el análisis de la llegada de la filosofía moderna a Colombia.

- Producción: se pone énfasis desmesurado en la generación de filosofía como un hecho cuantificable - número de textos, autores y obras - y la capacidad de esa producción de ser auténtica y útil. Estas consideraciones actúan en desmedro de la filosofía comprendida como el cultivo del saber humano que permite el pensamiento y la acción contextualizados, y, en definitiva, que es imprescindible para la vida y la acción filosófica.

En conclusión, la pregunta de si existe o no tradición filosófica en el país debe ser considerada innecesaria. Es un hecho que, en el territorio colombiano, a partir del siglo XIX ha habido intelectuales dedicados a reflexionar sobre los problemas de su época. De igual manera, se considera que la reconstrucción de esta tradición debe abarcar tanto estos primeros intentos como la introducción de la filosofía moderna en Colombia a partir de algunos personajes solitarios, como su posterior institucionalización en las 
facultades de filosofía. En cuanto a si se debería prescindir de la tradición filosófica colombiana, Pachón afirma:

Pienso que toda tradición es un subsuelo intelectual (para usar también una metáfora geológica) que no es monolítico, es variable, se transforma, se enriquece y se empobrece con cada nueva mirada, con cada nueva epistemología y metodología con que nos acerquemos a ella. El problema es considerar que las tradiciones están dadas de una vez por todas, y que olvidemos, más bien, que estas pueden ser cuestionadas, reconstruidas, criticadas, ampliadas, etc. Tenemos, en fin, una relación dinámica con la tradición y con el pasado, y este, querámoslo o no, se constituye en ese subsuelo en el cual podemos pararnos para partir hacia o construir nuevos horizontes; por lo demás, la tradición tiene un gran valor para comprender la producción filosófica presente, al punto que no hay creación filosófica sin diálogo, discusión, refutación, etc., con la tradición filosófica misma, ya que no partimos nunca de la nada, del vacío. Así, pues, lo que hay que tener en cuenta es que estas tradiciones se renuevan más frecuentemente de lo que pensamos: no están marmolizadas. (Pachón, 2019, p. 301)

La postura adoptada es que no se debe medir la existencia de una tradición por la altura de sus logros, por su aporte al orden mundial (europeo), sino que es preciso considerarla como el patrimonio intelectual y cultural de una sociedad que necesita de la filosofía para pensarse y entender sus conflictos. Hablamos de la necesidad de un uso social y decolonial de la filosofía dentro de un orden concreto: en un Estado democrático débil, con profundas desigualdades sociales que han derivado en constantes conflictos internos expresados durante décadas en prácticas de violencia, razón por la cual se presenta como necesaria una labor reflexiva que permita plantear nuevas maneras de organización para el siempre inconcluso proyecto de nación en el que hemos vivido. Finalmente, reivindicar la posibilidad de una reconstrucción decolonial de la tradición filosófica colombiana que confronte los cánones normalizadores de la autonomía, institucionalidad, centralismo y producción es una tarea urgente y necesaria para garantizar la vitalidad de la filosofía y la acción política cuando más se requiere: en estos tiempos virológicamente convulsos, pero interesantes para la innovación y la creatividad. 


\section{Referencias}

Betancur, J. C. (2015). Para un análisis crítico del concepto de normalización filosófica. Universitas Philosophica, 32(65), 137-158.

Campis, R. J. (2006). Filosofía en Colombia: ¿hay o no hay tradición? Primer Congreso Colombiano de Filosofía (pp. 409-428). Bogotá: Sociedad Colombiana de Filosofía. Recuperado de https://www.researchgate.net/publication/253559423

Jaramillo, J. (1954). Tradición y problemas de la filosofía en Colombia. Ideas y Valores, 3(9-10), 58-82.

Jaramillo, J. (1977). Etapas de la filosofía en la historia colombiana. En J. Jaramillo Uribe (ed.), La personalidad histórica de Colombia y otros ensayos (pp. 33-53). Bogotá: Instituto Colombiano de Cultura.

Jaramillo, J. (1982). El pensamiento colombiano en el siglo XIX. Bogotá: Temis.

López, C. A. (2012). Normalización de la filosofía y filosofía latinoamericana en Colombia: vivencia de un proceso. Universitas Philosophica, 58(29), 309-327.

López, C. A. (2014). Gobiernos, modernidad y producción escrita en Colombia (1880-1930): la escritura como terreno común de los antagonismos. Desafíos, 26(2), 43-71.

Pachón, D. (2011). Estudios sobre el pensamiento colombiano. Bogotá: Desde Abajo.

Pachón, D. (2012). Historiografía, eurocentrismo y universalidad en Enrique Dussel. Ideas y Valores, 61(148), 37-58.

Pachón, D. (2019). Debate: López Jiménez, Carlos Arturo. El terreno común de la escritura. Una historia de la producción filosófica en Colombia 1892-1910. Ideas $y$ Valores, 68(170), 291-303.

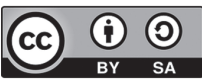

